\title{
EIGENFREQUENCIES OF THE NON-COLLINEARLY COUPLED EULER-BERNOULLI BEAM SYSTEM WITH DISSIPATIVE JOINTS
}

\author{
BY \\ WILLIAM H. PAULSEN \\ Department of Computer Science, Mathematics, and Physics, \\ Arkansas State University, State University, Arkansas
}

\begin{abstract}
In this paper, we will compute asymptotically the eigenfrequencies for the in-plane vibrations of the general non-collinear Euler-Bernoulli beam equation with dissipative joints. Many different kinds of dampers are allowed, even within the same structure. This generalizes a previous result for collinear structures. Matrix techniques are used to combine asymptotic analysis with the wave propagation method. We will find that if the lengths of the beams are rational, there will be a finite number of "streams" of eigenfrequencies, and, like the collinear case, each lies asymptotically to a vertical line.
\end{abstract}

1. Introduction. In the construction of large space structures, such as a large communication satellite or a space platform, different types of damping devices are commonly installed at the joints of the beams to suppress the vibrations. Without these damping devices, or dampers, small vibrations in the structure would persist indefinitely, or even slowly build up, which could lead to disastrous results. The joint and the damper together form what is called a dissipative joint. The structural stability of such a configuration of beams depends on the natural vibrations of that structure. Determining how well these vibrations are suppressed involves careful analysis. For NASA's proposed space platform, as many as 40 beams are required in order to make construction possible (information obtained at a NASA workshop).

Finding the exact values of the eigenfrequencies generally involves solving a highly complex transcendental equation. Thus, we will need to use asymptotics to find the first-order solutions. We will use the coupled beam structure, since it is the easiest type of structure to model mathematically. This structure is a chain formed by linking together two or more beams end to end, not necessarily collinearly. For now, we will work only with two-dimensional structures to find the in-plane vibrations, with hopes of extending this process to three dimensions in the future.

Received September 12, 1994.

1991 Mathematics Subject Classification. Primary 73D30, 73K12, 35P20.

Key words and phrases. Asymptotics, coupled beams, eigenfrequencies, Euler-Bernoulli, linear joints. 
2. General background. The Euler-Bernoulli beam equation, $m y_{t t}+E I y_{x x x x}=0$ with $0<x<L$ and $t>0$, is a standard mathematical model for a slender beam. Here $m$ denotes mass density per unit length, and $E I$ is the flexural rigidity of the beam. Appropriate boundary conditions and initial conditions will ensure a unique and stable solution [12]. Separation of variables can immediately be employed, giving

$$
\begin{gathered}
y(x, t)=T(t) \phi(x), \\
m T^{\prime \prime}=E I \lambda^{2} T, \\
\phi^{\prime \prime \prime \prime}+\lambda^{2} \phi=0 .
\end{gathered}
$$

The boundary conditions for the ends of the beam determine the eigenfrequencies $\lambda$. Each eigenfrequency has an associated eigenfunction $\phi_{\lambda_{j}}(x)$. The initial conditions can be decomposed into a linear combination of the eigenfunctions using an appropriate Green's function [12]. Thus, the problem is solvable provided that we can find the eigenfrequencies.

In $[5$, p. 1668], the case was considered in which two identical beams were linked together at an angle of 180 degrees by a certain type of dissipative joint, with one end of the structure fixed, and the other end free. Linear approximations were made for the angle displacement. Also, $m$ and $E I$ were assumed to be constant throughout the structure. In spite of these simplifications, the equations governing the eigenfrequencies were highly complex. However, they could be analyzed asymptotically by removing those terms that were small if the magnitude of the eigenfrequency was large. The spectrum of this structure was shown to be given by two sequences, or "streams" of eigenfrequencies,

$$
\lambda_{k} \sim \sqrt{E I / m}(4 k \pi-\pi)^{2}-E I \frac{k_{1}^{2} k_{2}^{2}+c_{1} c_{2}}{2 k_{1}^{2}} i
$$

and

$$
\lambda_{k} \sim \sqrt{E I / m}(4 k \pi+\pi)^{2}-\frac{4}{m k_{1}^{2}} i ; \quad \text { as } k \rightarrow \infty .
$$

Here, $k_{1}, k_{2}, c_{1}$, and $c_{2}$ are constants that depend on the damper in the joint.

In [13] the spectrum of the general $n$-beam collinear structure with the same assumptions was shown to have $n$ streams of eigenfrequencies, each of which lie asymptotically on a vertical line. This paper allowed three other designs of dissipative joints, and even different types of dampers within the same structure. The four types of dampers possible for collinearly connected beams are as follows, as mentioned in [5, p. 1667].

Type I: No external forces act on the joint, while the displacement vector and the beam coupling angle can change during dynamic vibrations.

Type II: The displacement vector is continuous across the joint, while the beam coupling angle can change. External forces may act on the joint, but only those that do not add a torque force.

Type III: The displacement vector and beam coupling angle are rigid, while any type of external force is allowed.

Type IV: The beam coupling angle is rigid, while the displacement vector can be discontinuous. The total external forces acting on the joint must be zero, but a torque force may remain. 


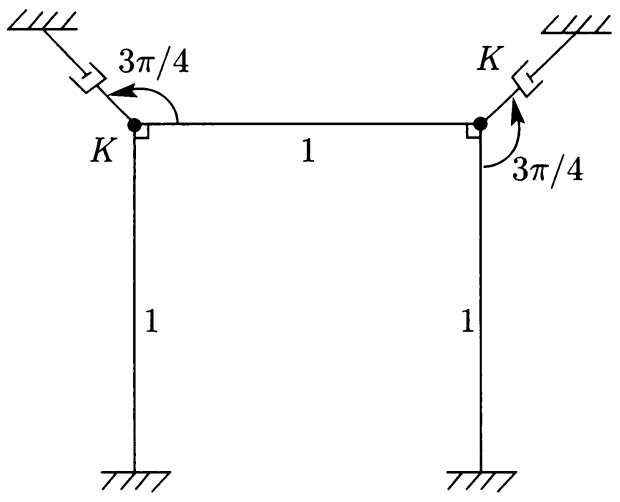

FIG. 1

The work in [13] also revealed a new way to compute the eigenfrequency "streams". For each beam in the structure, a 2-by-2 matrix was constructed containing the information about the length of the beam, and the type of damper at the end of that beam. These matrices then were multiplied together, including a fixed 1-by-2 matrix on the left and a 2-by-1 matrix on the right, to form a scalar function. The zeros of this function were the asymptotic approximations of the eigenfrequencies. However, this work only applied to collinearly coupled beams.

In a private communication with Chen and West, a specialized non-collinear 3-beam structure was analyzed, with the beams meeting at 90 degree angles with type III dampers at the joints, and the two ends of the structure fixed (see Fig. 1). Obtaining the equation for the eigenfrequencies involved taking the determinant of a 12-by-12 matrix. Using the Legendre-Tau spectral numerical method, the first few hundred eigenvalues could be computed, demonstrating that at least six streams of eigenfrequencies could be detected. From this, it was clear that if there was a method for solving the eigenfrequencies using matrices, as in [13], the matrices would have to be more complicated.

Later, Chen, along with Zhou [9], studied the vibrating string by decomposing the vibration waves into incident, reflected (including transmitted), and evanescent waves. By using the equations on how these waves were propagated, the eigenfrequencies could be computed. It was mentioned in this paper that the same method, called the wave propagation method (WPM), could be used for the Euler-Bernoulli beam equation, and one collinear example was given. This simplified the process, but some kind of asymptotics was still needed to solve the equations.

3. Fundamental ideas. In this paper, we will find a way to use matrices to determine asymptotically the in-plane vibrations for general non-collinear structures. That is, we will consider planar structures of $n$-beams, with dampers between the beams, as in Fig. 2 (see p. 440).

We will let $y^{j}(x, t), 0 \leq x \leq l_{j}, t \geq 0$ denote the transverse displacement function of the $j$ th beam. Since these are Euler-Bernoulli beams, the $y^{j}$ 's satisfy the PDE's

$$
m \frac{\partial^{2}}{\partial t^{2}} y^{j}(x, t)+E I \frac{\partial^{4}}{\partial x^{4}} y^{j}(x, t)=0, \quad \text { for } 0<x<l_{j}, t>0, j=1,2, \ldots, n .
$$




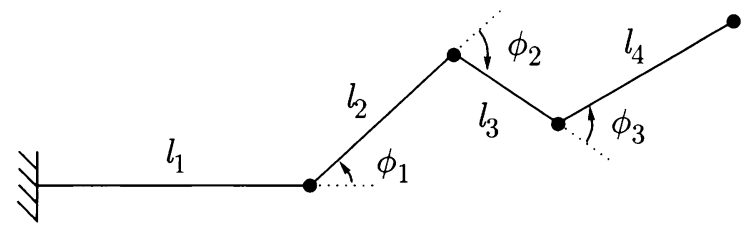

FIG. 2

Here, we use superscripts instead of subscripts to ease the notation of partial derivatives.

We will make three simplifying assumptions for this model:

(H1) We will only consider vibrations in the plane of the frame.

(H2) The beams are essentially non-compressible, that is, the change of length of the beams due to the forces exerted at the ends is negligible.

(H3) Forces exerted on a beam in the direction parallel to the length of the beam are propagated in a negligible amount of time.

Because of assumption ( $\mathrm{H} 2$ ), the longitudinal displacement is independent of the position within a given beam. Thus, we can denote the longitudinal displacement of the $j$ th beam by $z^{j}(t)$. Also, because of assumption (H3), the longitudinal force of a given beam depends only on time; so we can let $H^{j}(t)$ denote the longitudinal force of the $j$ th beam. Assumptions (H2) and (H3) together say that the longitudinal stiffness of the beams becomes "infinite". This is a common approach to structure problems and, in fact, is the basis of the displacement-stress approach for plates [10, p. 101ff].

For an Euler-Bernoulli beam, the relevant physical qualities are represented by:

$$
\begin{aligned}
& \text { Transverse Displacement }=y^{j}(x, t), \\
& \text { Longitudinal Displacement }=z^{j}(t), \\
& \text { Transverse Velocity }=y_{t}^{j}(x, t), \\
& \text { Longitudinal Velocity }=z_{t}^{j}(t), \\
& \text { Rotation }=y_{x}^{j}(x, t) \equiv \theta^{j}(x, t), \\
& \text { Angular Velocity }=y_{x t}^{j}(x, y)=\theta_{t}^{j}(x, t), \\
& \text { Bending Moment }=-E I y_{x x}^{j}(x, y) \equiv M^{j}(x, t), \\
& \text { Shear Force }=-E I y_{x x x}^{j}(x, y) \equiv V^{j}(x, t), \text { and } \\
& \text { Longitudinal Force }=H^{j}(x, t) .
\end{aligned}
$$

Let us begin by deriving a formula for the total energy in the system. The kinetic energy in each beam is given by

$$
\frac{1}{2} \int_{0}^{l_{j}} m\left(y_{t}^{j}\right)^{2}+m\left(z_{t}^{j}\right)^{2} d x .
$$

Also, the potential energy from the bending of each beam is

$$
\frac{1}{2} \int_{0}^{l_{j}} E I\left(y_{x x}^{j}\right)^{2} d x .
$$


So the total energy of the system is given by

$$
\mathcal{E}(t)=\sum_{j=1}^{n} \frac{1}{2} \int_{0}^{l_{j}} m\left(y_{t}^{j}\right)^{2}+m\left(z_{t}^{j}\right)^{2}+E I\left(y_{x x}^{j}\right)^{2} d x .
$$

Since the dampers are energy-dissipating, the rate of change of energy should always be negative. We can compute this change to be

$$
\frac{d}{d t} \mathcal{E}(t)=\sum_{j=1}^{n} \int_{0}^{l_{j}} m y_{t}^{j} y_{t t}^{j}+m z_{t}^{j} z_{t t}^{j}+E I y_{x x}^{j} y_{x x t}^{j} d x
$$

We can replace $m y_{t t}^{j}$ with $-E I y_{x x x x}^{j}$ to obtain

$$
\begin{aligned}
\frac{d}{d t} \mathcal{E}(t)= & \sum_{j=1}^{n} \int_{0}^{l_{j}}-E I y_{t}^{j} y_{x x x x}^{j}+m z_{t}^{j} z_{t t}^{j}+E I y_{x x}^{j} y_{x x t}^{j} d x \\
= & \sum_{j=1}^{n} \int_{0}^{l_{j}} \frac{\partial}{\partial x}\left(E I y_{x t}^{j} y_{x x}^{j}-E I y_{t}^{j} y_{x x x}^{j}+m x z_{t}^{j} z_{t t}^{j}\right) d x \\
= & \sum_{j=1}^{n} E I y_{x t}^{j}\left(l_{j}, t\right) y_{x x}^{j}\left(l_{j}, t\right)-E I y_{t}^{j}\left(l_{j}, t\right) y_{x x x}^{j}\left(l_{j}, t\right)+m l_{j} z_{t}^{j}(t) z_{t t}^{j}(t) \\
& -E I y_{x t}^{j}(0, t) y_{x x}^{j}(0, t)+E I y_{t}^{j}(0, t) y_{x x x}^{j}(0, t) .
\end{aligned}
$$

Because of assumption (H3), we have that

$$
H^{j}\left(l_{j}, t\right)=H^{j}(0, t)+m l_{j} z_{t t}^{j}(t)
$$

Hence, we can replace the term $m l_{j} z_{t}^{j}(t) z_{t t}^{j}(t)$ by $H^{j}\left(l_{j}, t\right) z_{t}^{j}(t)-H^{j}(0, t) z_{t}^{j}(t)$. We can also regroup the terms according to their position in the structure, giving

$$
\begin{gathered}
\frac{d}{d t} \mathcal{E}(t)=\theta_{t}^{1}(0, t) M^{1}(0, t)-y_{t}^{1}(0, t) V^{1}(0, t)-H^{1}(0, t) z_{t}^{1}(t)-\theta_{t}^{n}\left(l_{n}, t\right) M^{n}\left(l_{n}, t\right) \\
+y_{t}^{n}\left(l_{n}, t\right) V^{n}\left(l_{n}, t\right)+H^{n}\left(l_{n}, t\right) z_{t}^{n}(t) \\
+\sum_{j=1}^{n-1}\left(\theta_{t}^{j+1}(0, t) M^{j+1}(0, t)-y_{t}^{j+1}(0, t) V^{j+1}(0, t)-H^{j+1}(0, t) z_{t}^{j+1}(t)\right. \\
\left.\quad-\theta_{t}^{j}\left(l_{j}, t\right) M^{j}\left(l_{j}, t\right)+y_{t}^{j}\left(l_{j}, t\right) V^{j}\left(l_{j}, t\right)+H^{j}\left(l_{j}, t\right) z_{t}^{j}(t)\right) .
\end{gathered}
$$

If the beginning or end conditions are either free or clamped, the first six terms will be zero. The change in energy at each joint must be non-positive; so we have that

$$
\begin{aligned}
{\left[y_{t}^{j}\left(l_{j}, t\right) V^{j}\left(l_{j}, t\right)-y_{t}^{j+1}(0, t) V^{j+1}(0, t)\right] } & +\left[z_{t}^{j}(t) H^{j}\left(l_{j}, t\right)-z_{t}^{j+1}(t) H^{j+1}(0, t)\right] \\
& -\left[\theta_{t}^{j}\left(l_{j}, t\right) M^{j}\left(l_{j}, t\right)-\theta_{t}^{j+1}(0, t) M^{j+1}(0, t)\right] \leq 0 .
\end{aligned}
$$


One of the most important ideas needed to simplify the amount of calculations required for a given structure is to find a convenient basis for the propagating waves. Classically, the basis is chosen by letting the transverse displacement be

$$
y_{\lambda}^{j}(x, t)=\left(A_{j} e^{\eta x}+B_{j} e^{i \eta x}+C_{j} e^{-\eta x}+D_{j} e^{-i \eta x}\right) e^{\lambda t \sqrt{E I / m}} .
$$

However, we will find it convenient to consider another basis, using the following new functions:

$$
\begin{aligned}
& \operatorname{Hya}(x)=\frac{\cosh (x)+\cos (x)}{2}=\frac{e^{x}+e^{i x}+e^{-x}+e^{-i x}}{4} \\
& \operatorname{Hyb}(x)=\frac{\sinh (x)-\sin (x)}{2}=\frac{e^{x}+i e^{i x}-e^{-x}-i e^{-i x}}{4} \\
& \operatorname{Hyc}(x)=\frac{\cosh (x)-\cos (x)}{2}=\frac{e^{x}-e^{i x}+e^{-x}-e^{-i x}}{4} \\
& \operatorname{Hyd}(x)=\frac{\sinh (x)+\sin (x)}{2}=\frac{e^{x}-i e^{i x}-e^{-x}+i e^{-i x}}{4}
\end{aligned}
$$

One property of these "hybrid" functions is that the derivative of each one is the next, that is, -

$$
\begin{aligned}
\frac{d}{d x} \operatorname{Hya}(x) & =\operatorname{Hyb}(x) ; & \frac{d}{d x} \operatorname{Hyb}(x) & =\operatorname{Hyc}(x) ; \\
\frac{d}{d x} \operatorname{Hyc}(x) & =\operatorname{Hyd}(x) ; & \frac{d}{d x} \operatorname{Hyd}(x) & =\operatorname{Hya}(x) .
\end{aligned}
$$

We now can express the wave propagation in terms of the new basis:

$$
\begin{gathered}
y_{\lambda}^{j}(x, t)=\left(A_{j} \operatorname{Hya}(\eta x)+B_{j} \operatorname{Hyb}(\eta x)+C_{j} \operatorname{Hyc}(\eta x)+D_{j} \operatorname{Hyd}(\eta x)\right) e^{\lambda t \sqrt{E I / m}} \\
z_{\lambda}^{j}(t)=E_{j} e^{\lambda t \sqrt{E I / m}} ; \\
H_{\lambda}^{j}(0, t)=F_{j} E I \eta^{3} e^{\lambda t \sqrt{E I / m}} .
\end{gathered}
$$

Here, $\eta=\frac{1-i}{2} \sqrt{\lambda}$ so that $\lambda=i \eta^{2}$.

Another simplification is, rather than letting each matrix represent all of the information about one beam, including the angle between the beams and the type of damper at the end, we will let each matrix represent one element of the structure, such as a beam length, an angle between two beams, or a damper. For example, the above example by Chen and West could be described by the following:

Begin with a clamped end.

Put in a beam of length 1 .

Add a type III damper $45^{\circ}$ to the left.

Turn $90^{\circ}$ to the right.

Put in a beam of length 1 .

Add a type III damper $45^{\circ}$ to the left.

Turn $90^{\circ}$ to the right.

Put in a beam of length 1 .

Finish by clamping the end. 
Each line in the description will be represented by a separate matrix. So, in this case, there will be 9 matrices to multiply together. Although there are more matrices to multiply, each matrix will be simpler, sometimes even sparse.

We must consider how many different types of dampers there will be for non-collinearly coupled beams. For any given damper in the structure, either the transverse displacement or the shear force will be continuous. Likewise, either the longitudinal displacement or the longitudinal force will be continuous, and either the rotation or the bending moment will be continuous. Thus, there will be eight possible dampers for non-collinearly coupled beams, given by the following table:

$\begin{array}{ccc}\text { Type } & \text { Continuous variables } & \text { Discontinuous variables } \\ \text { I } & V, M, \text { and } H & y, \theta, \text { and } z \\ \text { II } & y, M \text {, and } z & V, \theta \text {, and } H \\ \text { III } & y, \theta \text {, and } z & V, M, \text { and } H \\ \text { IV } & V, \theta \text {, and } H & y, M, \text { and } z \\ \text { V } & y, M, \text { and } H & V, \theta, \text { and } z \\ \text { VI } & V, \theta \text {, and } z & y, M, \text { and } H \\ \text { VII } & V, M \text {, and } z & y, \theta \text {, and } H \\ \text { VIII } & y, \theta \text {, and } H & V, M, \text { and } z\end{array}$

The designs for types I, III, V, and VI are given in [5]. Also, a damper of type VII or VIII could be thought of as turning $90^{\circ}$ to the right, which exchanges the transverse and longitudinal displacement, adding a type V or VI damper, respectively, and finally turning $90^{\circ}$ to the left. Therefore, we only need to consider dampers of type I through VI.

4. Wave propagation matrices. We need to determine how a wave of the form (3.4) would propagate through the structure. We will do this by first finding the equations associated with each element of the structure. These then are converted to matrices that represent how the vector $\mathbf{v}=\left(A_{i}, B_{i}, C_{i}, D_{i}, E_{i}, F_{i}\right)$ is transformed by that element.

We begin by considering the two ends of the structure. When the beginning end of the first beam is fixed, the boundary conditions are given by $y^{1}(0, t)=y_{x}^{1}(0, t)=z^{1}(t)=0$. Likewise, when the terminal end of the last beam is free, the boundary conditions are $y_{x x}^{n}\left(l_{n}, t\right)=y_{x x x}^{n}\left(l_{n}, t\right)=H^{n}(t)=0$. Should the terminal end of the last beam be fixed, as in the example by [8], these boundary conditions are replaced by $y^{n}\left(l_{n}, t\right)=y_{x}^{n}\left(l_{n}, t\right)=$ $z^{n}(t)=0$.

The boundary conditions at each dissipative joint will depend on the type of damper involved. Figure 3 (see p. 444) demonstrates a type III damper, which is the easiest to design. A single dashpot with a damping coefficient of $K_{j}$ is attached to the joint at a distance of $r_{j}$ from the center of the joint. The angle from the next beam to the dashpot is given by $\gamma_{j}$. If we draw a line from the point of contact of the dashpot to the center of the joint, the angle from the dashpot to this line is given by $\delta_{j}$.

The boundary conditions for this damper can now be computed. We will consider only linear approximations to the angle displacement. The displacement vector and rotation are continuous across the damper, giving us three of the six boundary equations: 


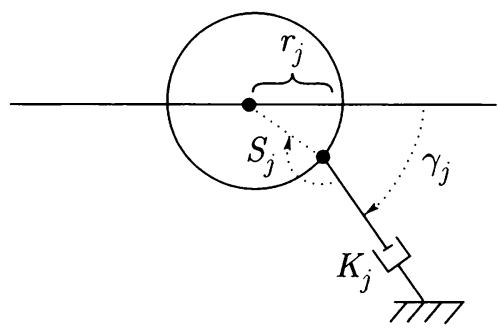

FIG. 3

$\theta^{j+1}(0, t)=\theta^{j}\left(l_{j}, t\right), y^{j+1}(0, t)=y^{j}\left(l_{j}, t\right)$, and $z^{j+1}(t)=z^{j}(t)$. For the other three equations, we first simplify the notation a bit by letting

$$
\begin{aligned}
a & =K_{j} \sin ^{2} \gamma_{j} / \sqrt{m E I} \\
b & =K_{j} \cos \gamma_{j} \sin \gamma_{j} / \sqrt{m E I} \\
c & =K_{j} r_{j} \sin \delta_{j} \sin \gamma_{j} / \sqrt{m E I} \\
d & =K_{j} \cos ^{2} \gamma_{j} / \sqrt{m E I} \\
e & =K_{j} r_{j} \sin \delta_{j} \cos \gamma_{j} / \sqrt{m E I} \\
f & =K_{j} r_{j}^{2} \sin ^{2} \delta_{j} / \sqrt{m E I} .
\end{aligned}
$$

The last three boundary conditions then become

$$
\begin{aligned}
& V^{j+1}(0, t)=V^{j}\left(l_{j}, t\right)+a \sqrt{m E I} y_{t}^{j}\left(l_{j}, t\right)+b \sqrt{m E I} z_{t}^{j}(t)+c \sqrt{m E I} \theta_{t}^{j}\left(l_{j}, t\right), \\
& H^{j+1}(0, t)=H^{j}\left(l_{j}, t\right)+b \sqrt{m E I} y_{t}^{j}\left(l_{j}, t\right)+d \sqrt{m E I} z_{t}^{j}(t)+e \sqrt{m E I} \theta_{t}^{j}\left(l_{j}, t\right), \\
& M^{j+1}(0, t)=M^{j}\left(l_{j}, t\right)-c \sqrt{m E I} y_{t}^{j}\left(l_{j}, t\right)-e \sqrt{m E I} z_{t}^{j}(t)-f \sqrt{m E I} \theta_{t}^{j}\left(l_{j}, t\right) .
\end{aligned}
$$

For a general damper of type III with more than one dashpot, the boundary conditions will still be the same, except that the constants $a, b, \ldots, f$ will be the sum of the corresponding constants for each dashpot. However, there still will be conditions that govern these constants, determined by the restriction that the damper is energy dissipative.

To determine this restriction, we substitute the six boundary equations into (3.3). We find that the rate of change of energy across the damper is given by

$$
-\sqrt{m E I}\left(y_{t}^{j+1}(0, t) \quad z_{t}^{j+1}(t) \quad \theta_{t}^{j+1}(0, t)\right)\left(\begin{array}{lll}
a & b & c \\
b & d & e \\
c & e & f
\end{array}\right)\left(\begin{array}{c}
y_{t}^{j+1}(0, t) \\
z_{t}^{j+1}(t) \\
\theta_{t}^{j+1}(0, t)
\end{array}\right) .
$$

Since the damper is energy-dissipating, the rate of change of energy across the damper must be non-positive. Thus we have that the matrix

$$
L_{j}=\left(\begin{array}{lll}
a & b & c \\
b & d & e \\
c & e & f
\end{array}\right)
$$


is a positive symmetric matrix, i.e.,

$$
\mathbf{w} L_{j} \mathbf{w}^{t} \geq 0 \text { for all vectors } \mathbf{w} \in \mathbb{R}^{3} \text {. }
$$

We call $L_{j}$ the constant matrix for the damper.

For brevity, we will let $\mathbf{v}_{j}=\left(A_{j}, B_{j}, C_{j}, D_{j}, E_{j}, F_{j}\right) \in \mathbb{R}^{6}$ be the components of the wave in terms of the new basis after the $j$ th line in the description. Then all six equations can be written succinctly as $\eta \mathbf{v}_{j+1}=\mathbf{v}_{j} \cdot M_{j}^{\mathrm{III}}$, where

$$
M_{j}^{\mathrm{III}}=\left(\begin{array}{cccccc}
\eta & -i a & i c \eta & 0 & 0 & i b \\
0 & \eta & 0 & 0 & 0 & 0 \\
0 & 0 & \eta & 0 & 0 & 0 \\
0 & -i c \eta & i f \eta^{2} & \eta & 0 & i e \eta \\
0 & -i b & i e \eta & 0 & \eta & i d \\
0 & 0 & 0 & 0 & 0 & \eta
\end{array}\right) .
$$

We can write similar matrices, which represent other types of dampers. For a type I damper, the boundary conditions can be written as

$$
\begin{gathered}
V^{j+1}=V^{j} ; \quad H^{j+1}=H^{j} ; \quad M^{j+1}=M^{j} ; \\
y_{t}^{j+1}=y_{t}^{j}+\frac{a}{\sqrt{m E I}} V^{j}+\frac{b}{\sqrt{m E I}} H^{j}+\frac{c}{\sqrt{m E I}} M^{j} \\
z_{t}^{j+1}=z_{t}^{j}+\frac{b}{\sqrt{m E I}} V^{j}+\frac{d}{\sqrt{m E I}} H^{j}+\frac{e}{\sqrt{m E I}} M^{j} \\
\theta_{t}^{j+1}=\theta_{t}^{j}-\frac{c}{\sqrt{m E I}} V^{j}-\frac{e}{\sqrt{m E I}} H^{j}-\frac{f}{\sqrt{m E I}} M^{j}
\end{gathered}
$$

Then the change of energy across the damper is

$$
-\frac{1}{\sqrt{m E I}}\left(V^{j+1}(0, t) \quad H^{j+1}(0, t) \quad M^{j+1}(0, t)\right)\left(\begin{array}{lll}
a & b & c \\
b & d & e \\
c & e & f
\end{array}\right)\left(\begin{array}{c}
V^{j+1}(0, t) \\
H^{j+1}(0, t) \\
M^{j+1}(0, t)
\end{array}\right) \leq 0 .
$$

By labeling the variables as we did, (4.1) holds for this type of damper as well. In fact, by labeling the variables of the dampers properly, Eq. (4.1) will hold for all dampers.

The boundary conditions for a type II damper are given by

$$
\begin{aligned}
y^{j+1} & =y^{j} ; \quad z^{j+1}=z^{j} ; \quad M^{j+1}=M^{j} \\
V^{j+1} & =V^{j}+a \sqrt{m E I} y_{t}^{j}+b \sqrt{m E I} z_{t}^{j}+c M^{j} \\
H^{j+1} & =H^{j}+b \sqrt{m E I} y_{t}^{j}+d \sqrt{m E I} z_{t}^{j}+e M^{j} \\
\theta_{t}^{j+1} & =\theta_{t}^{j}-c y_{t}^{j}-e z_{t}^{j}-\frac{f}{\sqrt{m E I}} M^{j}
\end{aligned}
$$


The boundary conditions for a type IV damper are

$$
\begin{aligned}
V^{j+1} & =V^{j} ; \quad H^{j+1}=H^{j} ; \quad \theta^{j+1}=\theta^{j} ; \\
y_{t}^{j+1} & =y_{t}^{j}+\frac{a}{\sqrt{m E I}} V^{j}+\frac{b}{\sqrt{m E I}} H^{j}+c \theta^{t} ; \\
z_{t}^{j+1} & =z_{t}^{j}+\frac{b}{\sqrt{m E I}} V^{j}+\frac{d}{\sqrt{m E I}} H^{j}+e \theta_{t}^{j} ; \\
M^{j+1} & =M^{j}-c V^{j}-e H^{j}-f \sqrt{m E I} \theta_{t}^{j} .
\end{aligned}
$$

The boundary conditions for a type $\mathrm{V}$ damper are

$$
\begin{gathered}
y^{j+1}=y^{j} ; \quad H^{j+1}=H^{j} ; \quad M^{j+1}=M^{j} ; \\
V^{j+1}=V^{j}+a \sqrt{m E I} y_{t}^{j}+b H^{j}+c M^{j} ; \\
z_{t}^{j+1}=z_{t}^{j}+b y_{t}^{j}+\frac{d}{\sqrt{m E I}} H^{j}+\frac{e}{\sqrt{m E I}} M^{j} \\
\theta_{t}^{j+1}=\theta_{t}^{j}-c y_{t}^{j}-\frac{e}{\sqrt{m E I}} H^{j}-\frac{f}{\sqrt{m E I}} M^{j} .
\end{gathered}
$$

The boundary conditions for a type VI damper are

$$
\begin{gathered}
V^{j+1}=V^{j} ; \quad z^{j+1}=z^{j} ; \quad \theta^{j+1}=\theta^{j} ; \\
y_{t}^{j+1}=y_{t}^{j}+\frac{a}{\sqrt{m E I}} V^{j}+b z_{t}^{j}+c \theta^{t} ; \\
H^{j+1}=H^{j}+b V^{j}+d \sqrt{m E I} z_{t}^{j}+e \sqrt{m E I} \theta_{t}^{j} \\
M^{j+1}=M^{j}-c V^{j}-e \sqrt{m E I} z_{t}^{j}-f \sqrt{m E I} \theta_{t}^{j} .
\end{gathered}
$$

Then the corresponding matrices $M_{j}$ for the other five types of dampers are given by

$$
\begin{aligned}
& M_{j}^{\mathrm{I}}=\left(\begin{array}{cccccc}
\eta & 0 & 0 & 0 & 0 & 0 \\
i a \eta^{2} & \eta & 0 & -i c \eta & i b \eta^{2} & 0 \\
i c \eta & 0 & \eta & -i f & i e \eta & 0 \\
0 & 0 & 0 & \eta & 0 & 0 \\
0 & 0 & 0 & 0 & \eta & 0 \\
-i b \eta^{2} & 0 & 0 & i e \eta & -i d \eta^{2} & \eta
\end{array}\right), \quad M_{j}^{\mathrm{II}}=\left(\begin{array}{cccccc}
\eta & -i a & 0 & -c & 0 & i b \\
0 & \eta & 0 & 0 & 0 & 0 \\
0 & c & \eta & -i f & 0 & -e \\
0 & 0 & 0 & \eta & 0 & 0 \\
0 & -i b & 0 & -e & \eta & i d \\
0 & 0 & 0 & 0 & 0 & \eta
\end{array}\right) \\
& M_{j}^{\mathrm{IV}}=\left(\begin{array}{cccccc}
1 & 0 & 0 & 0 & 0 & 0 \\
i a \eta & 1 & -c \eta & 0 & i b \eta & 0 \\
0 & 0 & 1 & 0 & 0 & 0 \\
c \eta & 0 & i f \eta & 1 & e \eta & 0 \\
0 & 0 & 0 & 0 & 1 & 0 \\
-i b \eta & 0 & e \eta & 0 & -i d \eta & 1
\end{array}\right), \quad M_{j}^{\mathrm{V}}=\left(\begin{array}{cccccc}
\eta & -i a & 0 & -c & b \eta & 0 \\
0 & \eta & 0 & 0 & 0 & 0 \\
0 & c & \eta & -i f & i e \eta & 0 \\
0 & 0 & 0 & \eta & 0 & 0 \\
0 & 0 & 0 & 0 & \eta & 0 \\
0 & -b \eta & 0 & i e \eta & -i d \eta^{2} & \eta
\end{array}\right), \\
& \text { and } M_{j}^{\mathrm{VI}}=\left(\begin{array}{cccccc}
\eta & 0 & 0 & 0 & 0 & 0 \\
i a \eta^{2} & \eta & -c \eta^{2} & 0 & 0 & -b \eta \\
0 & 0 & \eta & 0 & 0 & 0 \\
c \eta^{2} & 0 & i f \eta^{2} & \eta & 0 & i e \eta \\
b \eta & 0 & i e \eta & 0 & \eta & i d \\
0 & 0 & 0 & 0 & 0 & \eta
\end{array}\right)
\end{aligned}
$$


Notice that there are patterns between some of these matrices. We have that

$$
M_{j}^{\mathrm{III}}=-\eta^{2} M_{j}^{\mathrm{I}}(-1 / \eta)^{\mathrm{T}}, \quad M_{j}^{\mathrm{IV}}=-\eta M_{j}^{\mathrm{II}}(-1 / \eta)^{\mathrm{T}}, \quad M_{j}^{\mathrm{VI}}=-\eta^{2} M_{j}^{\mathrm{V}}(-1 / \eta)^{\mathrm{T}} .
$$

We will take advantage of these patterns later.

The matrix for turning an angle $\phi$ is given by

$$
M_{j}^{\text {angle }}=\left(\begin{array}{cccccc}
\cos \phi & 0 & 0 & 0 & \sin \phi & 0 \\
0 & \cos \phi & 0 & 0 & 0 & -\sin \phi \\
0 & 0 & 1 & 0 & 0 & 0 \\
0 & 0 & 0 & 1 & 0 & 0 \\
-\sin \phi & 0 & 0 & 0 & \cos \phi & 0 \\
0 & \sin \phi & 0 & 0 & 0 & \cos \phi
\end{array}\right)
$$

The matrix for a single beam of length $l$ is given by

$$
M_{j}^{\text {length }}=\left(\begin{array}{cccccc}
\operatorname{Hya}(l \eta) & \operatorname{Hyd}(l \eta) & \operatorname{Hyc}(l \eta) & \operatorname{Hyb}(l \eta) & 0 & 0 \\
\operatorname{Hyb}(l \eta) & \operatorname{Hya}(l \eta) & \operatorname{Hyd}(l \eta) & \operatorname{Hyc}(l \eta) & 0 & 0 \\
\operatorname{Hyc}(l \eta) & \operatorname{Hyb}(l \eta) & \operatorname{Hya}(l \eta) & \operatorname{Hyd}(l \eta) & 0 & 0 \\
\operatorname{Hyd}(l \eta) & \operatorname{Hyc}(l \eta) & \operatorname{Hyb}(l \eta) & \operatorname{Hya}(l \eta) & 0 & 0 \\
0 & 0 & 0 & 0 & 1 & 0 \\
0 & 0 & 0 & 0 & 0 & 1
\end{array}\right) .
$$

Finally, the two ends of the structure need to be converted into matrices. If the final end of the structure is either clamped or free, the boundary conditions can be put in the form

$$
\mathbf{v}_{n} \cdot M_{n}=\mathbf{0}
$$

where

$$
M_{n}^{\text {clamp }}=\left(\begin{array}{ccc}
1 & 0 & 0 \\
0 & 0 & 0 \\
0 & 0 & 0 \\
0 & 1 & 0 \\
0 & 0 & 1 \\
0 & 0 & 0
\end{array}\right) \quad \text { and } \quad M_{n}^{\text {free }}=\left(\begin{array}{ccc}
0 & 0 & 0 \\
1 & 0 & 0 \\
0 & 1 & 0 \\
0 & 0 & 0 \\
0 & 0 & 0 \\
0 & 0 & 1
\end{array}\right)
$$

Other types of end boundary conditions can be obtained by combining a damper with an end. For example, the boundary control used in [12] can be obtained by considering a type III damper immediately followed by a free end.

By combining all of the matrices together, we get the equation

$$
\mathbf{v}_{1} \cdot\left(M_{1} \cdot M_{2} \cdot M_{3} \cdots M_{n}\right)=\mathbf{0} \text {. }
$$

This gives us three equations with six unknowns. The other three equations come from the fact that the beginning end is clamped, which can be put in the form $\mathbf{v}_{1} \cdot M_{n}^{\text {clamp }}=\mathbf{0}$. This says that $A_{1}=D_{1}=E_{1}=0$; so only the second, third, and sixth columns of $\left(M_{1} \cdot M_{2} \cdot M_{3} \cdots M_{n}\right)$ will be important. We can express this by letting

$$
M_{0}^{\text {clamp }}=\left(\begin{array}{cccccc}
0 & 1 & 0 & 0 & 0 & 0 \\
0 & 0 & 1 & 0 & 0 & 0 \\
0 & 0 & 0 & 0 & 0 & 1
\end{array}\right) .
$$


Then there will be a nontrivial solution to the wave equations if and only if

$$
\operatorname{det}\left(M_{0} \cdot M_{1} \cdot M_{2} \cdots M_{n}\right)=0
$$

Let us denote $G=M_{0} \cdot M_{1} \cdot M_{2} \cdots M_{n}$. Then whenever $\eta$ is a solution to $|G|=0, \lambda=i \eta^{2}$ will be an eigenfrequency of the structure.

At this point, the work we have done is similar to the wave propagation method in [9]. We are essentially determining how six different types of waves are propagating through the structure, but the WPM is disguised in this setting because of our unusual basis. Yet this basis will help us later in taking the asymptotic estimates.

5. Asymptotic estimates. So far, we have found a way to compute the exact eigenfrequencies of the linear equations. However, for any nontrivial structure, the roots of the determinant in (4.2) will be almost impossible to obtain. Thus, asymptotic approximations seem reasonable. To solve for $\eta$ to a sufficient degree of approximation, we will need to estimate (4.2) to the highest two orders of $\eta$, as seen in [5].

Unfortunately, the highest orders of $\eta$ cancel as we take the determinant of $G$. Rather than trying to keep even more orders of $\eta$, we will try a different approach. We would like to be able to take the determinants of each matrix, causing the highest powers of $\eta$ to cancel before they were multiplied together. We can do this, by converting to larger matrices.

We can think of a 6-by-6 matrix as a linear function $F_{*}: V_{6} \rightarrow V_{6}$. This induces a linear map on the set of all tensors $F^{*}: \mathcal{T}\left(V_{6}\right) \rightarrow \mathcal{T}\left(V_{6}\right)$. In particular, $F^{*}$ maps every alternating covariant tensor of order 3 onto itself; thus, $F^{*}: \Lambda^{3}\left(V_{6}\right) \rightarrow \Lambda^{3}\left(V_{6}\right)$. This induction map is an anti-homomorphism, that is, if $H_{*}=F_{*} \circ G_{*}$, then $H^{*}=G^{*} \circ F^{*}$. See [2, p. 204] for details. By combining this transformation with a transpose, we can create a suitable homomorphism.

What this transformation amounts to is this: There are 20 ways to pick 3 rows out of the six, and 20 ways of picking 3 columns in the matrix. We can consider all 400 ways of forming a 3-by-3 submatrix from the original, and take the determinants of all 400 matrices, forming a 20-by-20 matrix $N_{j}$. Then finding the determinant of $G$ is the same as finding the product of the $N_{j}$ 's. The $3 \times 6$ and $6 \times 3$ matrices convert to $1 \times 20$ and $20 \times 1$ matrices.

We will consider the following basis for $\Lambda^{3}\left(V_{6}\right)$ :

$$
\begin{array}{llll}
\lambda_{1}=\omega_{1} \wedge \omega_{2} \wedge \omega_{3} & \lambda_{6}=\omega_{1} \wedge \omega_{3} \wedge \omega_{5} & \lambda_{11}=\omega_{1} \wedge \omega_{2} \wedge \omega_{6} & \lambda_{16}=\omega_{3} \wedge \omega_{4} \wedge \omega_{6} \\
\lambda_{2}=\omega_{1} \wedge \omega_{2} \wedge \omega_{4} & \lambda_{7}=\omega_{1} \wedge \omega_{4} \wedge \omega_{5} & \lambda_{12}=\omega_{1} \wedge \omega_{3} \wedge \omega_{6} & \lambda_{17}=\omega_{1} \wedge \omega_{5} \wedge \omega_{6} \\
\lambda_{3}=\omega_{1} \wedge \omega_{3} \wedge \omega_{4} & \lambda_{8}=\omega_{2} \wedge \omega_{3} \wedge \omega_{5} & \lambda_{13}=\omega_{1} \wedge \omega_{4} \wedge \omega_{6} & \lambda_{18}=\omega_{2} \wedge \omega_{5} \wedge \omega_{6} \\
\lambda_{4}=\omega_{2} \wedge \omega_{3} \wedge \omega_{4} & \lambda_{9}=\omega_{2} \wedge \omega_{4} \wedge \omega_{5} & \lambda_{14}=\omega_{2} \wedge \omega_{3} \wedge \omega_{6} & \lambda_{19}=\omega_{3} \wedge \omega_{5} \wedge \omega_{6} \\
\lambda_{5}=\omega_{1} \wedge \omega_{2} \wedge \omega_{5} & \lambda_{10}=\omega_{3} \wedge \omega_{4} \wedge \omega_{5} & \lambda_{15}=\omega_{2} \wedge \omega_{4} \wedge \omega_{6} & \lambda_{20}=\omega_{4} \wedge \omega_{5} \wedge \omega_{6}
\end{array}
$$

This basis determines the ordering of the elements of $N_{j}$. For example, the entry in the 9 th row and the 16th column of $N_{j}$ is given by taking the determinant of the submatrix obtained from the 2 nd, 4 th and 5 th rows, and 3 rd, 4 th and 6 th columns of $G_{j}$. Using 
this pattern, we can compute the matrices $N_{j}$. The end matrices $N_{0}$ and $N_{n}$ are the easiest to compute:

$$
\begin{aligned}
N_{0}^{\text {clamp }} & =(0,0,0,0,0,0,0,0,0,0,0,0,0,1,0,0,0,0,0,0), \\
N_{n}^{\text {clamp }} & =(0,0,0,0,0,0,1,0,0,0,0,0,0,0,0,0,0,0,0,0)^{\mathrm{T}}, \\
N_{n}^{\text {free }} & =(0,0,0,0,0,0,0,0,0,0,0,0,0,1,0,0,0,0,0,0)^{\mathrm{T}} .
\end{aligned}
$$

To display $N_{j}^{\text {angle }}$, we will let $\kappa=\cos \phi$ and $\sigma=\sin \phi$. Then $N_{j}^{\text {angle }}=$

$$
\left(\begin{array}{cccccccccccccccccccc}
\kappa^{2} & 0 & 0 & 0 & 0 & 0 & 0 & \kappa \sigma & 0 & 0 & 0 & \kappa \sigma & 0 & 0 & 0 & 0 & 0 & 0 & -\sigma^{2} & 0 \\
0 & \kappa^{2} & 0 & 0 & 0 & 0 & 0 & 0 & \kappa \sigma & 0 & 0 & 0 & \kappa \sigma & 0 & 0 & 0 & 0 & 0 & 0 & -\sigma^{2} \\
0 & 0 & \kappa & 0 & 0 & 0 & 0 & 0 & 0 & \sigma & 0 & 0 & 0 & 0 & 0 & 0 & 0 & 0 & 0 & 0 \\
0 & 0 & 0 & \kappa & 0 & 0 & 0 & 0 & 0 & 0 & 0 & 0 & 0 & 0 & 0 & -\sigma & 0 & 0 & 0 & 0 \\
0 & 0 & 0 & 0 & \kappa & 0 & 0 & 0 & 0 & 0 & 0 & 0 & 0 & 0 & 0 & 0 & \sigma & 0 & 0 & 0 \\
0 & 0 & 0 & 0 & 0 & 1 & 0 & 0 & 0 & 0 & 0 & 0 & 0 & 0 & 0 & 0 & 0 & 0 & 0 & 0 \\
0 & 0 & 0 & 0 & 0 & 0 & 1 & 0 & 0 & 0 & 0 & 0 & 0 & 0 & 0 & 0 & 0 & 0 & 0 & 0 \\
-\kappa \sigma & 0 & 0 & 0 & 0 & 0 & 0 & \kappa^{2} & 0 & 0 & 0 & -\sigma^{2} & 0 & 0 & 0 & 0 & 0 & 0 & -\kappa \sigma & 0 \\
0 & -\kappa \sigma & 0 & 0 & 0 & 0 & 0 & 0 & \kappa^{2} & 0 & 0 & 0 & -\sigma^{2} & 0 & 0 & 0 & 0 & 0 & 0 & -\kappa \sigma \\
0 & 0 & -\sigma & 0 & 0 & 0 & 0 & 0 & 0 & \kappa & 0 & 0 & 0 & 0 & 0 & 0 & 0 & 0 & 0 & 0 \\
0 & 0 & 0 & 0 & 0 & 0 & 0 & 0 & 0 & 0 & \kappa & 0 & 0 & 0 & 0 & 0 & 0 & -\sigma & 0 & 0 \\
-\kappa \sigma & 0 & 0 & 0 & 0 & 0 & 0 & -\sigma^{2} & 0 & 0 & 0 & \kappa^{2} & 0 & 0 & 0 & 0 & 0 & 0 & -\kappa \sigma & 0 \\
0 & -\kappa \sigma & 0 & 0 & 0 & 0 & 0 & 0 & -\sigma^{2} & 0 & 0 & 0 & \kappa^{2} & 0 & 0 & 0 & 0 & 0 & 0 & -\kappa \sigma \\
0 & 0 & 0 & 0 & 0 & 0 & 0 & 0 & 0 & 0 & 0 & 0 & 0 & 1 & 0 & 0 & 0 & 0 & 0 & 0 \\
0 & 0 & 0 & 0 & 0 & 0 & 0 & 0 & 0 & 0 & 0 & 0 & 0 & 0 & 1 & 0 & 0 & 0 & 0 & 0 \\
0 & 0 & 0 & \sigma & 0 & 0 & 0 & 0 & 0 & 0 & 0 & 0 & 0 & 0 & 0 & \kappa & 0 & 0 & 0 & 0 \\
0 & 0 & 0 & 0 & -\sigma & 0 & 0 & 0 & 0 & 0 & 0 & 0 & 0 & 0 & 0 & 0 & \kappa & 0 & 0 & 0 \\
0 & 0 & 0 & 0 & 0 & 0 & 0 & 0 & 0 & 0 & \sigma & 0 & 0 & 0 & 0 & 0 & 0 & \kappa & 0 & 0 \\
-\sigma^{2} & 0 & 0 & 0 & 0 & 0 & 0 & \kappa \sigma & 0 & 0 & 0 & \kappa \sigma & 0 & 0 & 0 & 0 & 0 & 0 & \kappa^{2} & 0 \\
0 & -\sigma^{2} & 0 & 0 & 0 & 0 & 0 & 0 & \kappa \sigma & 0 & 0 & 0 & \kappa \sigma & 0 & 0 & 0 & 0 & 0 & 0 & \kappa^{2}
\end{array}\right)
$$

To display $N_{j}^{\text {length }}$, we need to break the 20-by-20 matrix up into smaller pieces. Fortunately there is a pattern that we can utilize. First, let us introduce the variables

$$
\begin{aligned}
V & =\operatorname{Hya}(l \eta) ; \\
U & =\operatorname{Hyb}(l \eta) ; \\
V & =\operatorname{Hyc}(l \eta) ; \\
W & =\operatorname{Hyd}(l \eta) .
\end{aligned}
$$

If we let $A$ be the $4 \times 4$ matrix

$$
A=\left(\begin{array}{llll}
\operatorname{Hya}(l \eta) & \operatorname{Hyd}(l \eta) & \operatorname{Hyc}(l \eta) & \operatorname{Hyb}(l \eta) \\
\operatorname{Hyb}(l \eta) & \operatorname{Hya}(l \eta) & \operatorname{Hyd}(l \eta) & \operatorname{Hyc}(l \eta) \\
\operatorname{Hyc}(l \eta) & \operatorname{Hyb}(l \eta) & \operatorname{Hya}(l \eta) & \operatorname{Hyd}(l \eta) \\
\operatorname{Hyd}(l \eta) & \operatorname{Hyc}(l \eta) & \operatorname{Hyb}(l \eta) & \operatorname{Hya}(l \eta)
\end{array}\right),
$$

and $B$ be the $6 \times 6$ matrix

$$
B=\left(\begin{array}{cccccc}
T^{2}-U W & T W-U V & T V-U^{2} & W^{2}-T V & V W-T U & V^{2}-U W \\
T U-V W & T^{2}-V^{2} & T W-U V & T W-U V & W^{2}-U^{2} & V W-T U \\
T V-W^{2} & T U-V W & T^{2}-U W & U W-V^{2} & T W-U V & T V-U^{2} \\
U^{2}-T V & T U-V W & U W-V^{2} & T^{2}-U W & T W-U V & W^{2}-T V \\
U V-T W & U^{2}-W^{2} & T U-V W & T U-V W & T^{2}-V^{2} & T W-U V \\
V^{2}-U W & U V-T W & T V-W^{2} & U^{2}-T V & T U-V W & T^{2}-U W
\end{array}\right),
$$


then

$$
N_{j}^{\text {length }}=\left(\begin{array}{c|c|c|c}
A & 0 & 0 & 0 \\
\hline 0 & B & 0 & 0 \\
\hline 0 & 0 & B & 0 \\
\hline 0 & 0 & 0 & A
\end{array}\right) .
$$

To display the large matrices for the dampers, we will also need to introduce some new variables. Let

$$
\begin{array}{cl}
t=b e-c d, & r=a d-b^{2}, \\
u=b c-a e, & g=a f-c^{2}, \\
s=c e-b f, & v=d f-e^{2}, \\
w=a d f+2 b e c-c^{2} d-e^{2} a-b^{2} f .
\end{array}
$$

Then, after dividing by a factor of $\eta, N_{j}^{\mathrm{I}}=$

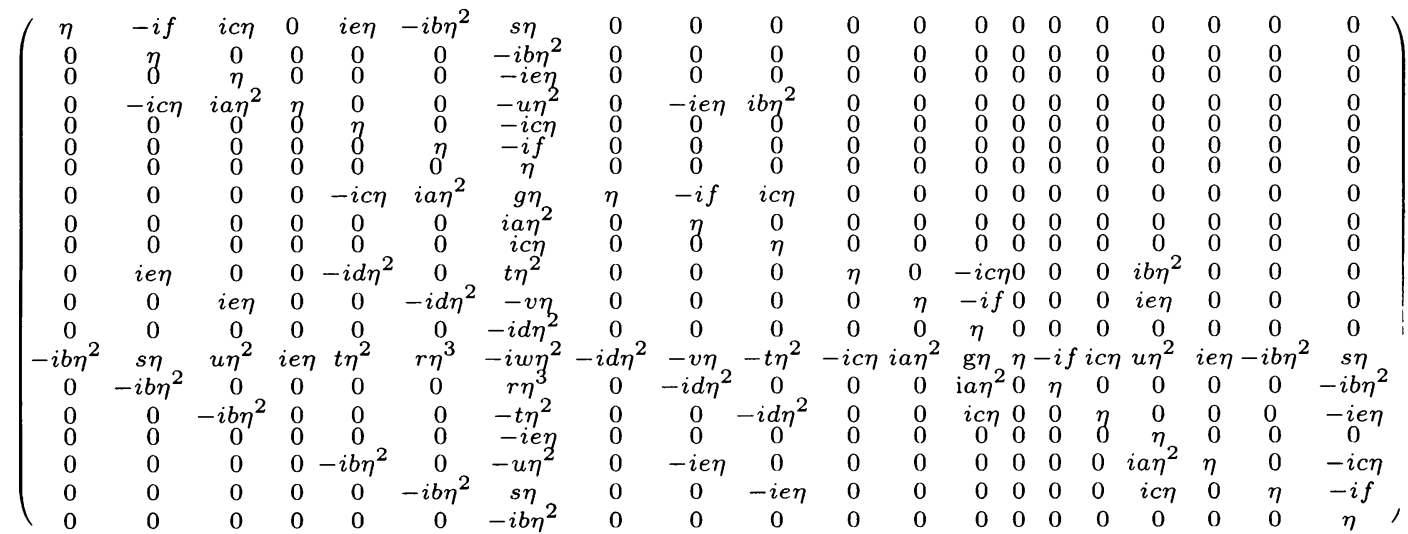

$N_{j}^{\mathrm{II}}=$

$\begin{array}{ccccccccccccccccccccc}\eta^{3} & -i f \eta^{2} & 0 & -c \eta^{2} & 0 & 0 & 0 & 0 & 0 & 0 & -e \eta^{2} & 0 & 0 & i b \eta^{2} & -s \eta & 0 & 0 & 0 & 0 & 0 \\ 0 & \eta^{3} & 0 & 0 & 0 & 0 & 0 & 0 & 0 & 0 & 0 & 0 & 0 & 0 & i b \eta^{2} & 0 & 0 & 0 & 0 & 0 \\ 0 & c \eta^{2} & \eta^{3} & -i a \eta^{2} & 0 & 0 & 0 & 0 & 0 & 0 & 0 & 0 & e \eta^{2} & 0 & i u \eta & i b \eta^{2} & 0 & 0 & 0 & 0 \\ 0 & 0 & 0 & \eta^{3} & 0 & 0 & 0 & 0 & 0 & 0 & 0 & 0 & 0 & 0 & e \eta^{2} & 0 & 0 & 0 & 0 & 0 \\ 0 & -e \eta^{2} & 0 & 0 & \eta^{3} & 0 & 0 & 0 & c \eta^{2} & 0 & i d \eta^{2} & 0 & 0 & 0 & -i t \eta & 0 & 0 & i b \eta^{2} & 0 & 0 \\ i b \eta^{2} & -s \eta & -e \eta^{2} & -i u \eta & c \eta^{2} & \eta^{3} & -i f \eta^{2} & -i a \eta^{2} & -g \eta & c \eta^{2} & -i t \eta & i d \eta^{2} & v \eta & r \eta & -i w & -i t \eta & e \eta^{2} & i u \eta & i b \eta^{2} & -\varepsilon \\ 0 & i b \eta^{2} & 0 & 0 & 0 & 0 & \eta^{3} & 0 & -i a \eta^{2} & 0 & 0 & 0 & i d \eta^{2} & 0 & r \eta & 0 & 0 & 0 & 0 & i b r \\ 0 & 0 & 0 & -e \eta^{2} & 0 & 0 & 0 & \eta^{3} & -i f \eta^{2} & 0 & 0 & 0 & 0 & i d \eta^{2} & v \eta & 0 & 0 & e \eta^{2} & 0 & 0 \\ 0 & 0 & 0 & 0 & 0 & 0 & 0 & 0 & \eta^{3} & 0 & 0 & 0 & 0 & 0 & i d \eta^{2} & 0 & 0 & 0 & 0 & 0 \\ 0 & 0 & 0 & -i b \eta^{2} & 0 & 0 & 0 & 0 & c \eta^{2} & \eta^{3} & 0 & 0 & 0 & 0 & -i t \eta & i d \eta^{2} & 0 & 0 & 0 & -e \\ 0 & 0 & 0 & 0 & 0 & 0 & 0 & 0 & 0 & 0 & \eta^{3} & 0 & 0 & 0 & c \eta^{2} & 0 & 0 & 0 & 0 & c \\ 0 & 0 & 0 & 0 & 0 & 0 & 0 & 0 & 0 & 0 & c \eta^{2} & \eta^{3} & -i f \eta^{2} & -i a \eta^{2} & -g \eta & c \eta^{2} & 0 & 0 & 0 & 0 \\ 0 & 0 & 0 & 0 & 0 & 0 & 0 & 0 & 0 & 0 & 0 & 0 & \eta^{3} & 0 & -i a \eta^{2} & 0 & 0 & 0 & 0 & 0 \\ 0 & 0 & 0 & 0 & 0 & 0 & 0 & 0 & 0 & 0 & 0 & 0 & 0 & \eta^{3} & -i f \eta^{2} & 0 & 0 & 0 & 0 & 0 \\ 0 & 0 & 0 & 0 & 0 & 0 & 0 & 0 & 0 & 0 & 0 & 0 & 0 & 0 & \eta^{3} & 0 & 0 & 0 & 0 & 0 \\ 0 & 0 & 0 & 0 & 0 & 0 & 0 & 0 & 0 & 0 & 0 & 0 & 0 & 0 & c \eta^{2} & \eta^{3} & 0 & 0 & 0 & 0 \\ 0 & 0 & 0 & 0 & 0 & 0 & 0 & 0 & 0 & 0 & -i b \eta^{2} & 0 & -e \eta^{2} & 0 & -i u \eta & 0 & \eta^{3} & -i a \eta^{2} & 0 & -c \\ 0 & 0 & 0 & 0 & 0 & 0 & 0 & 0 & 0 & 0 & 0 & 0 & 0 & 0 & -e \eta^{2} & 0 & 0 & \eta^{3} & 0 \\ 0 & 0 & 0 & 0 & 0 & 0 & 0 & 0 & 0 & 0 & 0 & 0 & 0 & i b \eta^{2} & -s \eta & -e \eta^{2} & 0 & c \eta^{2} & \eta^{3} & -i \\ 0 & 0 & 0 & 0 & 0 & 0 & 0 & 0 & 0 & 0 & 0 & 0 & 0 & 0 & i b \eta^{2} & 0 & 0 & 0 & 0 & \eta^{*}\end{array}$

$N_{j}^{\mathrm{III}}=-\eta^{3} N_{j}^{\mathrm{I}}(-1 / \eta)^{\mathrm{T}}$, 


$$
N_{j}^{\mathrm{IV}}=-\eta^{3} N_{j}^{\mathrm{II}}(-1 / \eta)^{\mathrm{T}},
$$

$N_{j}^{\mathrm{V}}=$

$\left(\begin{array}{cccccccccccccccccccc}\eta^{2} & -i f \eta & 0 & -c \eta & i e \eta^{2} & 0 & 0 & b \eta^{2} & i s \eta & 0 & 0 & 0 & 0 & 0 & 0 & 0 & 0 & 0 & 0 & 0 \\ 0 & \eta^{2} & 0 & 0 & 0 & 0 & 0 & 0 & b \eta^{2} & 0 & 0 & 0 & 0 & 0 & 0 & 0 & 0 & 0 & 0 & 0 \\ 0 & c \eta & \eta^{2} & -i a \eta & 0 & 0 & -i e \eta^{2} & 0 & u \eta & b \eta^{2} & 0 & 0 & 0 & 0 & 0 & 0 & 0 & 0 & 0 & 0 \\ 0 & 0 & 0 & \eta^{2} & 0 & 0 & 0 & 0 & -i e \eta^{2} & 0 & 0 & 0 & 0 & 0 & 0 & 0 & 0 & 0 & 0 & 0 \\ 0 & 0 & 0 & 0 & \eta^{2} & 0 & 0 & 0 & c \eta & 0 & 0 & 0 & 0 & 0 & 0 & 0 & 0 & 0 & 0 & 0 \\ 0 & 0 & 0 & 0 & c \eta & \eta^{2} & -i f \eta & -i a \eta & -g & c \eta & 0 & 0 & 0 & 0 & 0 & 0 & 0 & 0 & 0 & 0 \\ 0 & 0 & 0 & 0 & 0 & 0 & \eta^{2} & 0 & -i a \eta & 0 & 0 & 0 & 0 & 0 & 0 & 0 & 0 & 0 & 0 & 0 \\ 0 & 0 & 0 & 0 & 0 & 0 & 0 & \eta^{2} & -i f \eta & 0 & 0 & 0 & 0 & 0 & 0 & 0 & 0 & 0 & 0 & 0 \\ 0 & 0 & 0 & 0 & 0 & 0 & 0 & 0 & \eta^{2} & 0 & 0 & 0 & 0 & 0 & 0 & 0 & 0 & 0 & 0 & 0 \\ 0 & 0 & 0 & 0 & 0 & 0 & 0 & 0 & c \eta & \eta^{2} & 0 & 0 & 0 & 0 & 0 & 0 & 0 & 0 & 0 & 0 \\ 0 & i e \eta^{2} & 0 & 0 & -i d \eta^{3} & 0 & 0 & 0 & i t \eta^{2} & 0 & \eta^{2} & 0 & 0 & 0 & c \eta & 0 & 0 & -b \eta^{2} & 0 & 0 \\ b \eta^{2} & i s \eta & i e \eta^{2} & -u \eta & i t \eta^{2} & -i d \eta^{3} & -v \eta^{2} & -r \eta^{2} & i w \eta & i t \eta^{2} & c \eta \eta^{2} & -i f \eta-i a \eta & -g & c \eta i e \eta^{2} & -u \eta & -b \eta^{2} & -i s \eta \\ 0 & b \eta^{2} & 0 & 0 & 0 & 0 & -i d \eta^{3} & 0 & -r \eta^{2} & 0 & 0 & 0 & \eta^{2} & 0 & -i a \eta & 0 & 0 & 0 & 0 & -b \eta^{2} \\ 0 & 0 & 0 & i e \eta^{2} & 0 & 0 & 0 & -i d \eta^{3} & -v \eta^{2} & 0 & 0 & 0 & 0 & \eta^{2} & -i f \eta & 0 & 0 & i e \eta^{2} & 0 & 0 \\ 0 & 0 & 0 & 0 & 0 & 0 & 0 & 0 & -i d \eta^{3} & 0 & 0 & 0 & 0 & 0 & \eta^{2} & 0 & 0 & 0 & 0 & 0 \\ 0 & 0 & 0 & -b \eta^{2} & 0 & 0 & 0 & 0 & i t \eta^{2} & -i d \eta^{3} & 0 & 0 & 0 & 0 & c \eta & \eta^{2} & 0 & 0 & 0 & -i e \eta^{2} \\ 0 & 0 & 0 & 0 & b \eta^{2} & 0 & -i e \eta^{2} & 0 & u \eta & 0 & 0 & 0 & 0 & 0 & 0 & 0 & \eta^{2} & -i a \eta & 0 & -c \eta \\ 0 & 0 & 0 & 0 & 0 & 0 & 0 & 0 & -i e \eta^{2} & 0 & 0 & 0 & 0 & 0 & 0 & 0 & 0 & \eta^{2} & 0 & 0 \\ 0 & 0 & 0 & 0 & 0 & 0 & 0 & -b \eta^{2} & -i s \eta & -i e \eta^{2} & 0 & 0 & 0 & 0 & 0 & 0 & 0 & c \eta & \eta^{2} & -i f \eta \\ 0 & 0 & 0 & 0 & 0 & 0 & 0 & 0 & -b \eta^{2} & 0 & 0 & 0 & 0 & 0 & 0 & 0 & 0 & 0 & 0 & \eta^{2}\end{array}\right)$

and

$$
N_{j}^{\mathrm{VI}}=\eta^{3} N_{j}^{\mathrm{V}}(-1 / \eta)^{\mathrm{T}} .
$$

At this point, the equation $|G|=0$ can be replaced by the equation

$$
N_{0} \cdot N_{1} \cdot N_{2} \cdots N_{n}=0 .
$$

For convenience, let $S=N_{0} \cdot N_{1} \cdot N_{2} \cdots N_{n}$.

There is a method to all of this madness. Before, we had to keep many orders of $\eta$ to insure just one order of $\eta$ after taking the determinant. Now, we can approximate these matrices to find the first two orders of $\eta$ easily.

Since the complex conjugate of any eigenfrequency is another eigenfrequency, we will only consider eigenfrequencies $\lambda$ with nonnegative imaginary part. Also, the real part of $\lambda$ must be non-positive, since the dampers are all dissipative. Ignoring the false solution $\lambda=0$, we have that

$$
\pi / 2 \leq \arg (\lambda) \leq \pi
$$

Since $\lambda=i \eta^{2}, 0 \leq \arg (\eta) \leq \pi / 4$. We can use this to take estimates on the exponential powers of $\eta$. For example, $e^{l \eta} \gg e^{i l \eta}$ as $|\eta| \rightarrow \infty$. If we let $x=e^{i \eta}$, then we can get the first two orders of $\eta$ by using the following for $N_{j}^{\text {length }}$ (after multiplying by $8 e^{l(i \eta-\eta)}$ ):

$$
\widetilde{N}_{j}^{\text {length }}=\left(\begin{array}{c|c|c|c}
\widetilde{A} & 0 & 0 & 0 \\
\hline 0 & \widetilde{B} & 0 & 0 \\
\hline 0 & 0 & \widetilde{B} & 0 \\
\hline 0 & 0 & 0 & \widetilde{A}
\end{array}\right),
$$

where

$$
\widetilde{A}=\left(\begin{array}{llll}
2 x^{l} & 2 x^{l} & 2 x^{l} & 2 x^{l} \\
2 x^{l} & 2 x^{l} & 2 x^{l} & 2 x^{l} \\
2 x^{l} & 2 x^{l} & 2 x^{l} & 2 x^{l} \\
2 x^{l} & 2 x^{l} & 2 x^{l} & 2 x^{l}
\end{array}\right)
$$


and

$$
\widetilde{B}=\left(\begin{array}{cccccc}
x^{2 l}+1 & \left(\begin{array}{c}
(1-i) x^{2 l} \\
+1+i
\end{array}\right) & -i x^{2 l}+i & -i x^{2 l}+i & \left(\begin{array}{c}
(-1-i) x^{2 l} \\
-1+i
\end{array}\right) & -x^{2 l}-1 \\
\left(\begin{array}{c}
(1+i) x^{2 l} \\
+1-i
\end{array}\right) & 2 x^{2 l}+2 & \left(\begin{array}{c}
(1-i) x^{2 l} \\
+1+i
\end{array}\right) & \left(\begin{array}{c}
(1-i) x^{2 l} \\
+1+i
\end{array}\right) & -2 i x^{2 l}+2 i & \left(\begin{array}{c}
(-1-i) x^{2 l} \\
-1+i
\end{array}\right) \\
i x^{2 l}-i & \left(\begin{array}{c}
(1-i) x^{2 l} \\
+1-i
\end{array}\right) & x^{2 l}+1 & x^{2 l}+1 & \left(\begin{array}{c}
(1-i) x^{2 l} \\
+1+i
\end{array}\right) & -i x^{2 l}+i \\
i x^{2 l}-i & \left(\begin{array}{c}
(1+i) x^{2 l} \\
+1-i
\end{array}\right) & x^{2 l}+1 & x^{2 l}+1 & \left(\begin{array}{c}
(1-i) x^{2 l} \\
+1+i
\end{array}\right) & -i x^{2 l}+i \\
\left(\begin{array}{c}
(1-i) x^{2 l} \\
-1-i
\end{array}\right) & 2 i x^{2 l}-2 i & \left(\begin{array}{c}
(1-i) x^{2 l} \\
+1-i
\end{array}\right) & \left(\begin{array}{c}
(1+i) x^{2 l} \\
+1-i
\end{array}\right) & 2 x^{2 l}+2 & \left(\begin{array}{c}
(1-i) x^{2 l} \\
+1+i
\end{array}\right) \\
-x^{2 l}-1 & \left(\begin{array}{c}
(1-i) x^{2 l} \\
-1-i
\end{array}\right) & i x^{2 l}-i & i x^{2 l}-i & \left(\begin{array}{c}
(1+i) x^{2 l} \\
+1-i
\end{array}\right) & x^{2 l}+1
\end{array}\right)
$$

We can also simplify $N_{j}^{\mathrm{I}}$ through $N_{j}^{\mathrm{VI}}$ a bit by considering only the last two orders of $\eta$. In each instance, there may be up to three cases per type of damper.

The type I damper has three possibilities: either $r=a d-b^{2} \neq 0, r=0$ but $a+d \neq 0$, or $a=d=0$. This determines whether the highest power of $\eta$ in $N_{j}^{\mathrm{I}}$ is 3,2 , or 1 . We refer to the three possibilities as type Ia, type Ib, and type Ic dampers. The simplified matrices are given as $\tilde{N}_{j}^{\mathrm{Ia}}, \widetilde{N}_{j}^{\mathrm{Ib}}$, and $\widetilde{N}_{j}^{\mathrm{Ic}}$.

The type II damper must have an $\eta^{3}$ term. So there is only one way it could reduce. We refer to the simplified matrix as $\tilde{N}_{j}^{\mathrm{II}}$, which contains the terms of $N_{j}^{\mathrm{II}}$ of order $\eta^{2}$ and $\eta^{3}$.

The type III damper has two cases, one in which $f \neq 0$, which we call a type IIIa damper, and one in which $f=0$, called a type IIIb damper. Note that in a type IIIb damper, $c$ and $e$ must also be 0 , because of (4.1). We refer to the simplified matrices as $\tilde{N}_{j}^{\text {IIIa }}$ and $\tilde{N}_{j}^{\text {IIIb }}$.

The type IV damper will have three possibilities, depending on whether the matrix $L$ is of rank 3,2 , or 1 . The three cases will be called type IVa, type IVb, and type IVc respectively. The corresponding simplified matrices will be $\widetilde{N}_{j}^{\mathrm{IVa}}, \widetilde{N}_{j}^{\mathrm{IVb}}$, and $\widetilde{N}_{j}^{\mathrm{IVc}}$.

The type $\mathrm{V}$ damper will have two cases: either $d \neq 0$, called a type Va damper, or $d=0$, called a type $\mathrm{Vb}$ damper. The corresponding simplified matrices will be $\widetilde{N}_{j}^{\mathrm{Va}}$ and $\tilde{N}_{j}^{\mathrm{Vb}}$.

The type VI damper has three possibilities. Either $g=a f-c^{2} \neq 0$, giving a type VIa damper, $g=0$ but $a+f \neq 0$, called a type VIb damper, or $a=f=0$, called a type VIc damper. The corresponding simplified matrices are referred to as $\widetilde{N}_{j}^{\mathrm{VIa}}, \widetilde{N}_{j}^{\mathrm{VIb}}, \widetilde{N}_{j}^{\mathrm{IVc}}$.

Finally, we will let $\widetilde{N}_{j}^{\text {angle }}=N_{j}^{\text {angle }}$. Then (4.2) can be approximated to two orders of $\eta$ by considering the equation

$$
\widetilde{S}=\tilde{N}_{0} \cdot \tilde{N}_{1} \cdot \widetilde{N}_{2} \cdots \tilde{N}_{n}=0
$$

To compute the positions of the streams of the eigenvalues we proceed as in [13]. If the lengths are all integers, we can divide (5.1) by factors of $\eta$ to obtain an equation of the form

$$
f(x)+\frac{g(x)}{\eta}=O\left(\eta^{-2}\right)
$$


for some polynomial functions $f(x)$ and $g(x)$.

In order to get $\lambda=i \eta^{2}$ to first order, we need to solve (5.2) to second order in $\eta$, where $x=e^{i \eta}$. To first order, $x \sim r$, where $r$ is a root of $f(x)=0$. This gives $\eta \sim-i \log |r|+\arg (r)+2 k \pi$, where $k$ is an integer. We need one more order of $\eta$. So let us suppose that

$$
\eta \sim-i \log |r|+\arg (r)+2 k \pi+\frac{c}{k}+O\left(k^{-2}\right)
$$

for some $c$. Then

$$
e^{i \eta} \sim r e^{i c / k} \sim r\left(1+\frac{i c}{k}\right) \sim r+\frac{i c r}{k}+O\left(k^{-2}\right) .
$$

Then $f\left(e^{i \eta}\right) \sim f(r+i c / k) \sim i c r f^{\prime}(r) / k+O\left(k^{-2}\right)$, and $g\left(e^{i \eta}\right) \sim g(r)+O\left(k^{-1}\right)$. Since $\eta \sim 2 k \eta,(5.2)$ becomes

$$
\frac{i c r f^{\prime}(r)}{k}+\frac{g(r)}{2 k \pi} \sim O\left(k^{-2}\right)
$$

so that

$$
c=\frac{i g(r)}{2 \pi r f^{\prime}(r)} .
$$

This gives

$$
\eta \sim-i \log |r|+\arg (r)+2 k \pi+\frac{i g(r)}{2 \eta r f^{\prime}(r)}+O\left(k^{-2}\right)
$$

Then

$$
\lambda_{k}=i \eta^{2} \sim(\arg (r)+2 k \pi)^{2} i-\log ^{2}|r| i+2 \log |r|(\arg (r)+2 k \pi)-\frac{2 g(r)}{r f^{\prime}(r)}+O\left(k^{-1}\right) .
$$

If $|r|=1$, this reduces to

$$
\lambda_{k}=\frac{-2 g(r)}{r f^{\prime}(r)}+(\arg (r)+2 k \pi)^{2} i+O\left(k^{-1}\right) .
$$

In this case, the sequence of eigenfrequencies is called a linear stream. When $|r| \neq 1$, the sequence if called a parabolic stream, since the eigenfrequencies lie on a parabola.

6. All streams are linear. In this section, we will show that parabolic streams are impossible, regardless of the configuration. This amounts to showing that all roots of $f(x)$ in (5.2) have magnitude one. Let us first suppose that all of the beams have interer lengths, so that $f(x)$ is a polynomial.

LEMma 6.1. If $f(x)$ is a polynomial, and the leading coefficient of $f(x)$ has the same magnitude as the constant coefficient, then for all roots $r,|r|=1$.

Proof. Because the leading coefficient has the same magnitude as the constant coefficient, the product of all the roots of $f(x)$ must have magnitude 1 . So if any root does not have magnitude 1 , there will be some root for which $|r|>1$. Then there would be a sequence of solutions $\lambda_{k}$ to $|\widetilde{S}|=0$ such that $\operatorname{Re}\left(\lambda_{k}\right) \sim \log |r| \sqrt{\lambda_{k}}$ as $\lambda_{k} \rightarrow \infty$. But $\log |r|>0$; so the real part of these eigenvalues would be positive. Moreover, the approximations we made from $S$ to $\widetilde{S}$ are valid for $\frac{\pi}{2}-\gamma \leq \arg \lambda \leq \pi$ for some $\gamma>0$, as shown in [13]. Thus, $|G|=0$ would have a sequence of eigenfrequencies with positive real part. But this contradicts the fact that the dampers were chosen to be dissipative. Thus, parabolic streams will not exist. 
ThEOREM 6.2. For any configuration of the beams, and any combination of dampers, the roots of $f(x)$ will all have magnitude 1 . Thus, the eigenfrequencies will approach a finite number of streams, each lying asymptotically to a vertical line.

Proof. Let us first suppose that all beams are of integer length, so that $f(x)$ is a polynomial. From the above lemma, it is enough to show that the leading coefficient of $f(x)$ has the same magnitude as the constant coefficient. We will prove this by observing some patterns in the matrices $\widetilde{N}_{j}$. Notice that for each entry in $\widetilde{N}_{j}^{\text {length }}$, the leading coefficient in $x$ is the complex conjugate of the constant coefficient. We can express this relationship by the equation

$$
\bar{x}^{2 l} \widetilde{N}_{j}^{\text {length }}(1 / \bar{x})=\overline{\widetilde{N}_{j}^{\text {length }}(x)} .
$$

A similar relationship occurs in the other matrices, mainly because the other matrices do not depend on $x$. Thus, since $\widetilde{N}_{j}^{\text {angle }}$ is real,

$$
\widetilde{N}_{j}^{\text {angle }}(1 / \bar{x})=\overline{\widetilde{N}_{j}^{\text {angle }}(x)} .
$$

For the damping matrices, only the highest order of $\eta$ will contribute to $f(x)$. As long as the matrix is either purely real or purely complex to the highest order of $\eta$, we will have

$$
\widetilde{N}_{j}(1 / \bar{x}) \sim \pm \overline{\widetilde{N}_{j}(x)}
$$

This is the case when the damper is of type Ia, Ib, Ic, II, IIIa, IIIb, IVa, Va, Vb, VIa, or VIc. The only exceptions are types IVb, IVc, and VIb. We will deal with these three cases later.

Because of these patterns in the matrices, as long as there are no dampers of type IVb, IVc, or VIb, we have that

$$
\bar{x}^{d} f(1 / \bar{x})= \pm \overline{f(x)}
$$

where $d$ is the degree of $f(x)$. From this, we can conclude that the leading coefficient of $f(x)$ is plus or minus the complex conjugate of the constant coefficient. Thus, they will have the same magnitude.

Even though the matrices for the type IVb, IVc, and VIb dampers are not purely real or purely complex, the above pattern will be maintained after the matrix is multiplied on both sides by $\widetilde{N}^{\text {length }}$. The details are as follows.

Because for this setting we are only interested in the leading and constant coefficient in $x$, we can replace $\widetilde{N}_{j}^{\text {length }}$ by a simpler matrix,

$$
\widehat{N}_{j}^{\text {length }}=\left(\begin{array}{c|c|c|c}
0 & 0 & 0 & 0 \\
\hline 0 & \widetilde{B} & 0 & 0 \\
\hline 0 & 0 & \widetilde{B} & 0 \\
\hline 0 & 0 & 0 & 0
\end{array}\right) .
$$


Any time there is a damping matrix, it will be between two length matrices. However, there may be an angle matrix multiplied either before or after the damping matrix. Thus, the most general format for which the damping matrix could appear is of the form

$$
P=\widehat{N}_{j-2}^{\text {length }} \cdot \widetilde{N}_{j-1}^{\text {angle }} \cdot \widetilde{N}_{j}^{\text {damper }} \cdot \widetilde{N}_{j+1}^{\text {angle }} \cdot \widehat{N}_{j+2}^{\text {length }} .
$$

What is interesting is that, even though $\widetilde{N}_{j}^{\text {damper }}$ is neither purely real nor purely complex for dampers of type IVb, IVc, or VIb, the entire product in (6.1) still satisfies

$$
\bar{x}^{2 l_{j-2} 2 l_{j+2}} P(1 / \bar{x})=\overline{P(x)} \text {. }
$$

This has been verified with MATHEMATICA. Thus, the leading coefficient of $f(x)$ will still have the same magnitude as the constant coefficient, even when dampers of type IVb, IVc, and VIb are involved.

Next, let us consider the case where all the beams have rational length. Finding a lowest common denominator $d$, we can express each $l_{j}=m_{j} / d$. By letting $y=x^{1 / d}, x^{l_{j}}$ will equal $y^{m_{j}}$, so the equation $f(x)=0$ for $l_{j}=m_{j} / d$ will be the same as the equation $\tilde{f}(y)=0$, where $\tilde{f}(x)$ is the equation for the system with $l_{j}=m_{j}$. From the previous case, all solutions to $\tilde{f}(y)=0$ must have magnitude one, and since $x=y^{d}$, all solutions of $f(x)=0$ will have magnitude one.

Finally, there is the case where one or more of the beams have irrational length. We can approximate this case to arbitrary precision in the case for which the beam lengths are rational. Since the roots of the equation $f(x)=0$ have magnitude 1 for all such approximations, and $f(x)$ depends continuously on the parameters $l_{1}, l_{2}, \ldots, l_{n}$, then all solutions to $f(x)=0$ must have magnitude one when the beam lengths are irrational.

7. An example. We now can use the large matrices to find the approximate eigenfrequencies of Fig. 1. The two dampers are both of type IIIb, and we will let the matrix $L_{j}$, which describes the dampers, be given by

$$
L_{1}=L_{2}=\left(\begin{array}{ccc}
K / 2 & K / 2 & 0 \\
K / 2 & K / 2 & 0 \\
0 & 0 & 0
\end{array}\right) \text {. }
$$

After multiplying out the 9 matrices, and dividing by $\eta^{4}$, we find that (5.2) holds, with

$$
\begin{aligned}
f(x) & =-4\left((3+3 i) x^{6}-x^{5}+(3+i) x^{4}+2 x^{3}+(3-i) x^{2}-x+(3-3 i)\right) \\
& =-4(x+i)\left((1+i) x^{2}+x+(1-i)\right)\left(3 x^{3}-(2+i) x^{2}+(1+2 i) x-3 i\right)
\end{aligned}
$$

and $g(x)=-4 K\left(2 x^{6}+(2-3 i) x^{4}+(-2-3 i) x^{2}-2\right)$.

By substituting the six roots of $f(x)$ into (5.4), we have that the six streams of eigenfrequencies are approximately as follows:

$$
\begin{aligned}
\lambda_{1, k} & \sim 0+(2 \pi k-\pi / 2)^{2} i ; \\
\lambda_{2, k} & \sim 0+(2 \pi k-2.71756161)^{2} i ; \\
\lambda_{3, k} & \sim 0+(2 \pi k+1.14676529)^{2} i ; \\
\lambda_{4, k} & \sim-0.73654372 K+(2 \pi k-1.15536425)^{2} i ; \\
\lambda_{5, k} & \sim-0.54770276 K+(2 \pi k-4.04101037)^{2} i ; \\
\lambda_{6, k} & \sim-0.04908685 K+(2 \pi k+0.48398550)^{2} i .
\end{aligned}
$$


These results agree with the eigenfrequencies given in [8]. Notice that three of the six streams lie on the imaginary axis, making it appear as though there were only four streams of eigenfrequencies. The following table gives the exact values of the first three eigenfrequencies in each stream and compares these with the values given above. One can see a rapid convergence in each stream to the vertical line, especially when the real part of the stream is small. The exact values were computed using Newton's method on Eq. (4.2).

Eigenfrequency
$\lambda_{5,1}$
$\lambda_{2,1}$
$\lambda_{1,1}$
$\lambda_{4,1}$
$\lambda_{6,1}$
$\lambda_{3,1}$
$\lambda_{5,2}$
$\lambda_{2,2}$
$\lambda_{1,2}$
$\lambda_{4,2}$
$\lambda_{6,2}$
$\lambda_{3,2}$
$\lambda_{5,3}$
$\lambda_{2,3}$
$\lambda_{1,3}$
$\lambda_{4,3}$
$\lambda_{6,3}$
$\lambda_{3,3}$

$$
\begin{aligned}
& \text { Exact value } \\
& -.73638885+5.030004 i \\
& 12.648041 i \\
& 22.373285 i \\
& -.73219254+26.186625 i \\
& -.04889164+45.798548 i \\
& 55.198084 i \\
& -.54770525+72.662051 i \\
& 96.998715 i \\
& 120.903392 i \\
& -.73623564+130.189924 i \\
& -.04904502+170.310350 i \\
& 188.050075 i \\
& -.54756328+219.281537 i \\
& 260.241239 i \\
& -0.4908685+ \\
& -.54770276+ \\
& \begin{array}{lll}
-.73654372+130.211066 i & 0.03
\end{array} \\
& -.54770276+219.293021 i \quad 0.01 \\
& 298.555535 i \\
& -.73640152+313.070718 i \\
& -.04906775+373.784831 i \\
& 399.852861 i
\end{aligned}
$$$$
\text { Approximate value }
$$$$
-.54770276+5.027349 i
$$$$
12.713672 i \quad 0.1
$$$$
22.206610 i \quad 0.2
$$$$
-.73654372+26.294549 i \quad 0.1
$$$$
45.794601 i \quad 0.004
$$$$
55.204165 i \quad 0.006
$$$$
72.681768 i
$$$$
0.02
$$$$
96.999039 i \quad 0.0003
$$$$
120.902654 i \quad 0.001
$$$$
\begin{array}{lll}
-.04908685+170.311795 i & 0.001
\end{array}
$$$$
188.050096 i \quad 0.00002
$$$$
260.241240 i \quad 0.000001
$$

$$
\begin{array}{rl}
298.555533 i & 0.000002 \\
-.73654372+313.084419 i & 0.01 \\
-.04908685+373.785824 i & 0.001 \\
399.852862 i & 0.000001
\end{array}
$$

Much of the work in this paper required the use of the symbolic manipulator MATHEMATICA running on a SUN Microsystems workstation for the computation of the large matrices.

Although this paper analyzes a linear model of a physical system, the experimental data cited in [5] and [6] indicate that this model is an accurate one. However, we still are only considering vibrations that occur within the plane. Hopefully, analysis for structures that do not lie in a plane can be done using a similar technique, and this will be treated in a future work.

\section{REFERENCES}

[1] C. Aganovic and Z. Tutez, A justification of the one-dimensional model of an elastic beam, Math. Methods in Applied Sci. 8, 1-14 (1986)

[2] W. Boothby, An Introduction to Differentiable Manifolds and Riemannian Geometry, Academic Press, Inc., 1986

[3] G. Chen, M. C. Delfour, A. M. Krall, and G. Payne, Modeling, stabilization and control of serially connected beams, SIAM J. Control Optim. 25, 526-546 (1987)

[4] G. Chen, S. G. Krantz, D. W. Ma, C. E. Wayne, and H. H. West, The Euler-Bernoulli beam equation with boundary energy dissipation, Operator Methods for Optimal Control Problem, Marcel Dekker, New York, 1987, pp. 67-96 
[5] G. Chen, S. G. Krantz, D. L. Russell, C. E. Wayne, H. H. West, and M. P. Coleman, Analysis, designs and behavior of dissipative joints for coupled beams, SIAM J. Appl. Math. 49, 1665-1693 (1989)

[6] G. Chen, S. G. Krantz, D. L. Russell, C. E. Wayne, H. H. West, and J. Zhou, Modeling, analysis and testing of dissipative beam joints-experiments and data smoothing, Math. Comput. Modelling 11, 1011-1016 (1988)

[7] G. Chen and H. Wang, Asymptotic locations of eigenfrequencies of Euler-Bernoulli beam with nonhomogeneous structural and viscous damping coefficients, SIAM J. Control Optim. 29, 347367 (1991)

[8] G. Chen and H. H. West, private communication

[9] G. Chen and J. Zhou, The wave propagation method for the analysis of boundary stabilization in vibration structures, SIAM J. Appl. Math. 50, 1254-1283 (1990)

[10] P. G. Ciarlet, Plates and Junctions in Elastic Multi-Structures, Springer-Verlag, New York, 1990

[11] J. B. Keller and S. I. Rubinow, Asymptotic solution of eigenvalue problems, Ann. Physics 9, 24-75 (1960)

[12] A. M. Krall, Asymptotic Stability of the Euler-Bernoulli Beam with Boundary Control, J. Math. Anal. Appl. 137, 288-295 (1989)

[13] S. G. Krantz and W. Paulsen, Asymptotic eigenfrequency distributions for the N-beam EulerBernoulli coupled beam equation with dissipative joints, J. Symbolic Comp. 11, 369-418 (1991)

[14] W. D. Pilkey, Manual for the Response of Structural Members, Vol. I, Illinois Inst. of Tech. Res. Inst. Project J6094, Chicago, IL, 1969 